\title{
The quantum mechanics of electric conduction in crystals
}

\author{
Raina J. Olsen and Giovanni Vignale \\ Department of Physics, University of Missouri-Columbia, Columbia, Missouri 65211
}

(Received 11 December 2009; accepted 5 May 2010)

\begin{abstract}
We introduce a model of electrons incident on a one-dimensional periodic potential and show that conduction is a result of the interference of different parts of an electron wave that bounce multiple times through a series of potentials, ultimately being transmitted or reflected. Simple iterative equations are derived that determine the transmission and reflection coefficients of a periodic system. We find that total reflection of the electron occurs in certain energy ranges, as well as ranges of energy within which complete transmission can occur. The energy ranges in which transmission occurs are equivalent to the conduction bands traditionally found using Bloch's theorem. These equations are solved numerically, allowing a concrete demonstration of conduction. The disordered case is also addressed. (C) 2010 American Association of Physics Teachers.
\end{abstract}

[DOI: 10.1119/1.3436664]

\section{INTRODUCTION}

Interference occurs when two or more waves are superimposed, resulting in a new wave pattern. Historically, the hypothesis by De Broglie ${ }^{1}$ of the wavelike nature of electrons and subsequent experimental discovery of an electron diffraction pattern by Thomson et $a l^{2}$ was one of the great triumphs of quantum mechanics. In 1976 Merli et al. ${ }^{3}$ performed an experiment that created an interference pattern by sending electrons one at a time through a Young's double slit apparatus, proving that each electron can interfere with itself. While there is no agreed upon physical interpretation for the phase of a particle, there are many such experimental results that demonstrate its necessity, including the phenomenon of electrical conduction.

In this paper we introduce a model of electrons incident on a one-dimensional crystal whose behavior can be understood in terms of the interference of multiple pieces of an electron wave reflected and transmitted from a periodic potential barrier, each unit of which has a given reflection and transmission coefficient. The interference results in certain ranges of energy within which complete reflection of the electron occurs, as well as ranges of energy within which complete transmission can occur. These ranges occur regardless of the number of potential barriers in the periodic system. This behavior is in contrast to the classical case where the probability of transmission decreases monotonically as the number of potential barriers increases, and complete transmission can occur only when the transmission coefficient of each unit of the periodic potential is of magnitude one, thus highlighting the necessity of quantum mechanics in describing conduction. The energy ranges in which transmission occurs will be shown to be equivalent to the conduction bands traditionally found using Bloch's theorem, as in Ref. 4, Chap. 8.

This model has several advantages. Rather than using abstract theoretical concepts such as momentum space and periodic boundary conditions, the conduction of electrons is treated in real space as a scattering problem where the electrons are introduced at an interface to a finite size crystal and either transmitted or reflected. Additionally, the mathematics is simple with only algebra, the concept of complex numbers, and the geometric series needed for a student to entirely understand the model. The model also allows direct numeri- cal experimentation during which the students can see for themselves the emergence of conduction bands and the effects of disorder on the system.

\section{CLASSICAL SYSTEM}

In classical mechanics, assuming all of the properties of the system are perfectly known, one can predict exactly what will happen to any particle. The only source of uncertainty is the imperfect knowledge of the system. By introducing uncertainty, one can speak instead of the probability of various outcomes. If more than one route to a certain outcome is possible, the probabilities of each route are added to find the total probability of the outcome.

Consider a one-dimensional classical barrier that transmits incident electrons with a probability $T$ and reflects them with a probability $R$. This uncertainty is assumed to come about from imperfect knowledge of the system. Conservation of probability requires that $T+R=1$. Now consider a composite barrier composed of two of these barriers. We might naively calculate the transmission coefficient of the composite barrier to be $T^{2}$, which is the probability of transmitting through the first barrier and then through the second barrier. However, there are an infinite number of ways in which an electron can be transmitted through the composite barrier, as shown in Fig. 1. An electron might transmit through the first barrier, reflect off the second barrier, reflect off the first barrier, and then transmit through the second barrier. Likewise, an electron might transmit through the first barrier, reflect between the two barriers twice, and then transmit through the second barrier. All of these possibilities are accounted for in the infinite series

$$
\begin{aligned}
T_{2}= & T T+T R R T+T R R R R T+T R R R R R R T \\
& +T R R R R R R R R T+\ldots
\end{aligned}
$$

This geometric series can be summed exactly to give

$$
T_{2}=T^{2}\left(1+R^{2}+R^{4}+\ldots\right)=\frac{T^{2}}{1-R^{2}} .
$$

Another barrier can be added to the chain using the same procedure. The process can be repeated as many times as desired to find the transmission coefficient of a composite barrier composed of any finite number of barriers whose in- 
periodic potential

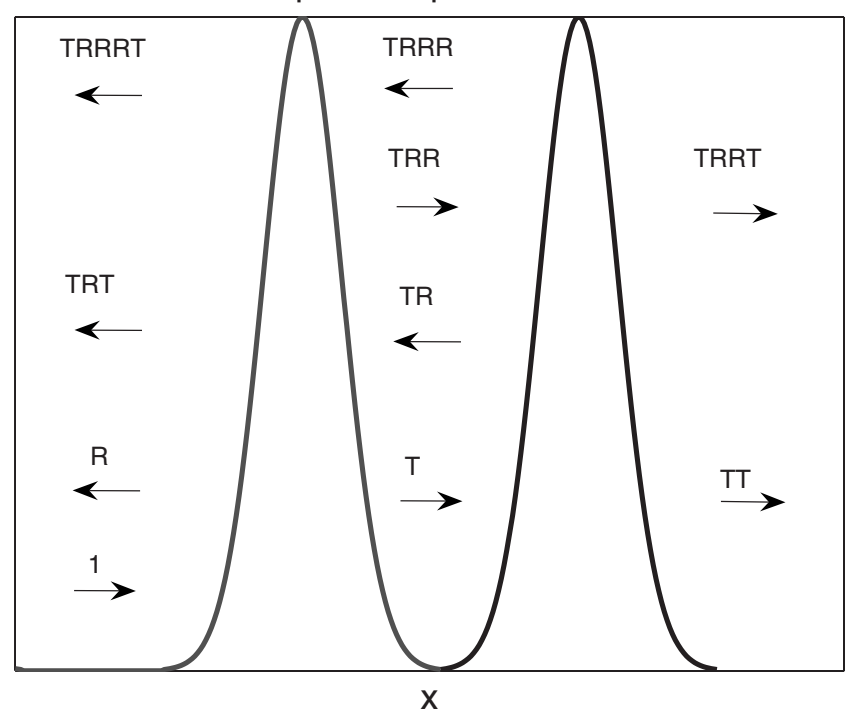

Fig. 1. An electron is incident upon a potential composed of two identical classical potentials. The probability of transmission can be found by adding the probabilities that the electron transmits through the first barrier, reflects multiple times between the barriers, and then transmits through the second barrier.

dividual probabilities of transmission and reflection are known. This process can be described recursively.

Consider a one-dimensional periodic barrier composed of $N$ identical barriers. If the probability of transmission $T_{N}$ and reflection $R_{N}$ through the entire chain are known and one more identical barrier is added to the end, as shown in Fig. 2, the probability of transmission through the new composite barrier can be calculated by adding together the probability of transmission through all of the barriers plus the probabil-

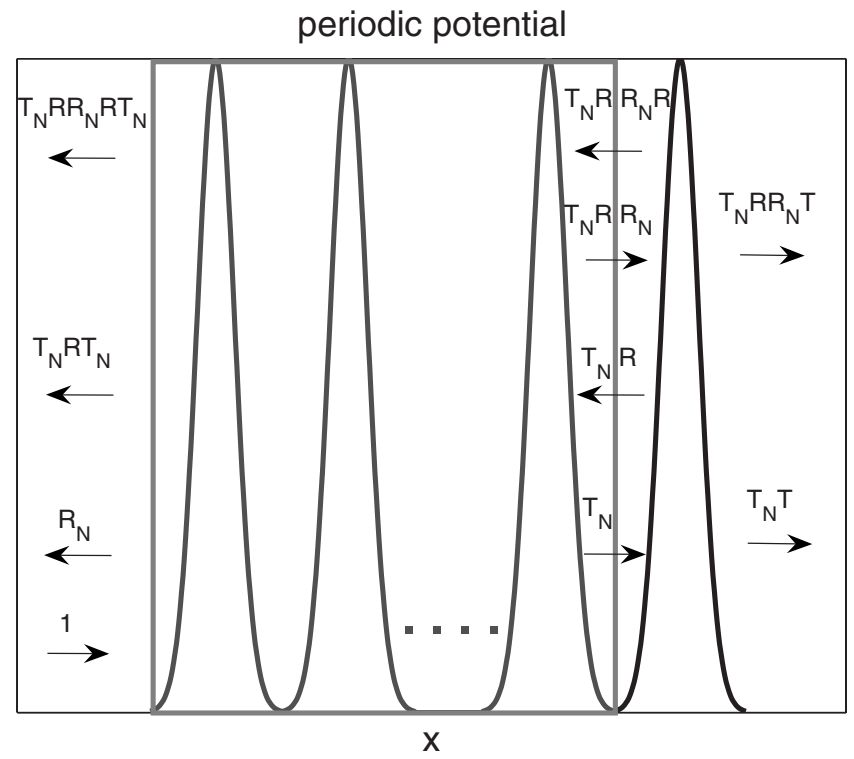

Fig. 2. An electron is incident upon a periodic potential composed of $N+1$ identical classical potentials. The probability of transmission can be found by adding the probabilities that the electron transmits through the first barrier (composed of the first $N$ potentials, enclosed in the gray box), reflects multiple times between the first barrier and the second barrier (composed of the one new potential), and then transmits through the second barrier. ity of transmitting through the original chain, reflecting multiple times between the original chain and the new barrier, and transmitting through the additional barrier,

$$
T_{N+1}=T_{N} T+T_{N} R R_{N} T+T_{N} R^{2} R_{N}^{2} T+\ldots=\frac{T_{N} T}{1-R_{N} R} .
$$

We let $R=1-T$ and $R_{N}=1-T_{N}$ and divide each term by $T T_{N}$ to find

$$
T_{N+1}=\frac{T_{N} T}{T_{N}+T-T_{N} T}=\frac{1}{(1 / T)+\left(1 / T_{N}\right)-1} .
$$

The inverse of Eq. (4) is

$$
\frac{1}{T_{N+1}}=\frac{1}{T}+\frac{1}{T_{N}}-1 \text {. }
$$

Equation (5) can be further simplified by noting that $1 / T_{N}-1=\left(1-T_{N}\right) / T_{N}=R_{N} / T_{N}$. The ratio $R_{N} / T_{N}$ goes to zero when all the electrons are transmitted and goes to infinity when all electrons are reflected; therefore this ratio is comparable to the resistance. By subtracting one from both sides and applying the same simplification, Eq. (5) becomes

$$
\frac{R_{N+1}}{T_{N+1}}=\frac{R_{N}}{T_{N}}+\frac{R}{T}=(N+1) \frac{R}{T},
$$

where the last equality follows by induction.

Except in the trivial case for which $T=1$ and $R=0$, meaning each barrier has no effect on an incident electron, Eq. (6) demonstrates that the resistance of a periodic barrier increases linearly with the number of composite barriers. A similar classical derivation of Ohm's law is given in Ref. 5, p. 62. If electrical conduction were a completely classical phenomenon, an electrical field would have to be applied to continually add energy to the electrons as they travel through a periodic barrier, and thus the resistance would be nonzero. This behavior fits our intuitive sense of how the world works. In most materials resistance, even if very small, is always nonzero. However, if we work out the resistance classically for a material such as pure copper, it turns out to be an order of magnitude larger than the measured value.

\section{QUANTUM SYSTEM}

The probability amplitude in quantum mechanics is a complex number, having both magnitude and phase. Its magnitude must be squared to find the probability of an outcome. If more than one route to a certain outcome is possible, the probability amplitudes rather than the probabilities are summed, and then the magnitude of the total probability amplitude is squared in the usual way to find the probability. Because the probability of an outcome is dependent only on the magnitude of the probability amplitude, there is no direct way to observe the phase of a particle. Phase is an eminently wavelike feature implying that there is some cyclical process all particles undergo, which is nearly hidden from view. The only way its existence can be demonstrated is through the phenomenon of interference. Interference occurs when complex probability amplitudes from multiple routes are added, resulting in a significantly higher or lower probability than would be expected classically.

The wave function of an electron incident on a potential barrier has a probability of being transmitted or reflected. 
The following relations between the probability amplitudes of the transmitted part of the wave, $t$, and the reflected part of the wave, $r$ are proven in Appendix A:

$$
\begin{aligned}
& |t|^{2}+|r|^{2}=1, \\
& t=|t| e^{i \theta}, \quad r= \pm i|r| e^{i \theta} .
\end{aligned}
$$

The sum of the probability of transmission $T=|t|^{2}$ and of reflection $R=|r|^{2}$ is one, as required by the conservation of probability. Equation (8) implies that the phase of the reflection coefficient is shifted by $\pm \pi / 2$ relative to the phase of the transmission coefficient.

Now consider a one-dimensional periodic potential composed of $N$ identical potential barriers whose centers are separated by a distance $a$. Each barrier has total transmission and reflection coefficients $\tilde{t}$ and $\widetilde{r}$. The total transmission coefficient includes the geometric phase gained by an electron with wave vector $k$ as it travels through a distance $a$ and is related to the transmission coefficient by $\tilde{t}=t e^{i k a}$. This relation is discussed in Appendix B. The same procedure will be used as for the classical case, except now the probability amplitudes are summed instead of the probabilities. If the transmission and reflection coefficients of the entire chain, $\widetilde{t}_{N}$ and $\widetilde{r}_{N}$, are known and one more potential barrier is added to the end of the chain, the transmission coefficient of the $N+1$ potential barriers can be calculated by adding together the part of the incoming wave that is transmitted through all of the barriers plus the parts of the incoming wave that are transmitted through the original chain, reflected multiple times between the original chain and new barrier, and then transmitted through the second barrier,

$$
\tilde{t}_{N+1}=\tilde{t}_{N} \tilde{t}+\widetilde{t}_{N} \tilde{r} \tilde{r}_{N} \tilde{t}+\widetilde{t}_{N} \widetilde{r}^{2} \widetilde{r}_{N}^{2} \tilde{t}+\ldots=\frac{\widetilde{t}_{N} \tilde{t}}{1-\widetilde{r}_{N} \tilde{r}} .
$$

Notice that Eq. (9) is almost the same as Eq. (3), except that probability amplitudes, rather than probabilities, are summed.

Likewise the reflection coefficient can be calculated by adding together the part of the incoming wave that is reflected from the original chain plus the parts of the incoming wave that transmit through the original chain, reflect between the original chain and the new barrier multiple times, and are transmitted back through the original chain,

$$
\widetilde{r}_{N+1}=\widetilde{r}_{N}+\tilde{t}_{N}^{2} \tilde{r}+\tilde{t}_{N}^{2} \tilde{r}^{2} \widetilde{r}_{N}+\ldots=\widetilde{r}_{N}+\frac{\widetilde{t}_{N}^{2} \widetilde{r}}{1-\tilde{r}_{N} \widetilde{r}} .
$$

By using Eqs. (9) and (10), it is possible to calculate the transmission and reflection coefficient of an infinite number of periodic potentials using the transmission coefficient of only one potential. This method can be shown to give identical results to the transfer matrix method outlined by $\mathrm{Kiang}^{7}$ and Griffiths et al. ${ }^{8}$

Equations (9) and (10) can be used to see how the behavior of an electron in a crystal is dependent on the magnitude or phase of $\tilde{t}$. Let us see under which conditions the magnitude of the transmission coefficient goes to zero as $N \rightarrow \infty$. Because of the conservation of probability, the magnitude of the reflection coefficient will also go to one as $N \rightarrow \infty$, and electrons will be completely reflected from the periodic potential.

From Eq. (10) it is clear that under these conditions, the second term on the right-hand side goes to zero, and thus $\tilde{r}_{\infty+1}=\widetilde{r}_{\infty}$ and the reflection coefficient approaches a value that is constant in phase as well as magnitude. Given Eq. (8), the transmission coefficient will also approach to a value that is constant in phase.

Equation (9) can be rewritten by using $\tilde{t}=|t| e^{i \theta}$, $\tilde{r}= \pm i|r| e^{i \theta}$, and $\lim _{N \rightarrow \infty} \widetilde{r}_{N}= \pm i e^{i \theta_{\infty}}$,

$$
\lim _{N \rightarrow \infty} \frac{t_{N+1}^{\prime}}{t_{N}^{\prime}}=\lim _{N \rightarrow \infty} \frac{\tilde{t}}{1-\tilde{r}_{N} \tilde{r}}=\frac{|t|}{e^{-i \theta} \pm|r| e^{i \theta_{\infty}}} .
$$

Because the phase of the transmission coefficient goes to a constant, the right-hand side of Eq. (11) must be real. The numerator, $|t|$, is already real. For the denominator to be real, the imaginary parts of $e^{-i \theta}$ and $\pm|r| e^{i \theta_{\infty}}$ must cancel. This leads to the condition

$$
\frac{\sin \theta}{|r|}= \pm \sin \theta_{\infty}
$$

Equation (12) can be satisfied only if the magnitude of the left-hand side of Eq. (12) is less than or equal to 1, meaning

$$
|\sin \theta| \leq|r| \text {. }
$$

This result is surprising. In the classical case, the transmission coefficient of a chain always goes to zero as the length of the chain approaches infinity, whereas in the quantum case, this behavior only sometimes holds. There are also some conditions where the transmission coefficient of an infinite chain is nonzero, which are given by the relation

$$
|\sin \theta|>|r| \text {. }
$$

By squaring Eq. (14), subtracting each side from one, and using the relation $|t|^{2}+|r|^{2}=1$, the condition can be written as

$$
|\cos \theta|^{2}<|t|^{2} \text {. }
$$

We take the square root, divide by $|t|$, and find

$$
\frac{|\cos \theta|}{|t|}<1
$$

Based on Eq. (16) we conclude that as the transmission coefficient varies, there are always bands in which the probability of transmission is nonzero, and band gaps in which the probability of transmission goes to zero as the number of potentials goes to infinity. At zero temperature every pure periodic potential is a perfect conductor with zero resistance to certain electrons. The widths of the bands depend on $|t|$. The larger the transmission coefficient, the wider are these allowed bands. Because $t$ typically increases with energy, these bands generally become wider as the energy increases. Figure 3 shows the transmission probability, calculated iteratively using Eq. (9), of an electron that is inside of a band or outside of a band. For comparison purposes, the same is shown for the classical case, calculated using Eq. (4). Note that in the quantum case when the energy is inside of a band, the function is periodic with the transmission probability repeatedly reaching one. In the quantum case where the energy is outside of a band, the probability drops off exponentially. The classical case is between these two extremes and the probability drops off as $1 / N$.

Equations (9) and (10) can also be used to see how the behavior of an electron in a crystal is dependent on the size of the crystal and the energy of the electron. Figure 4 shows a plot of the probability of transmission, $T=\left|t_{N}(k)\right|^{2}$ with 


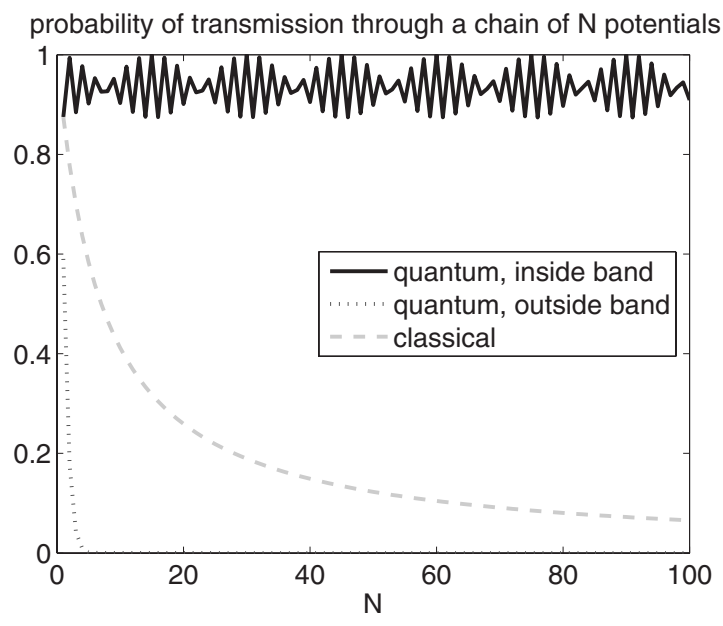

Fig. 3. Probability of transmission versus the number of identical periodic potentials for the classical and quantum cases, with the latter case being inside or outside of a band. In the classical case, the probability of transmission approaches zero, whereas in the quantum case, the probability repeats periodically for an energy inside the band and approaches zero exponentially for an energy outside the band. In all cases the probability of transmission through a single barrier is the same.

$N=3,6$, and 1000 for a delta-function potential with a given periodicity $a$. Inside of the allowed bands the probability of transmission is nonzero, with $N-1$ peaks in each band where complete transmission is achieved. This complete transmission occurs without applying any potential across the crystal, meaning that the resistance is zero for electrons in these $N-1$ energy states. Outside of the band, reflection occurs, with the resistance approaching $\infty$ as the number of potentials increases. As the wave vector $k$ (and thus the energy) increases, the width of the bands increases and the width of the band gaps decreases.

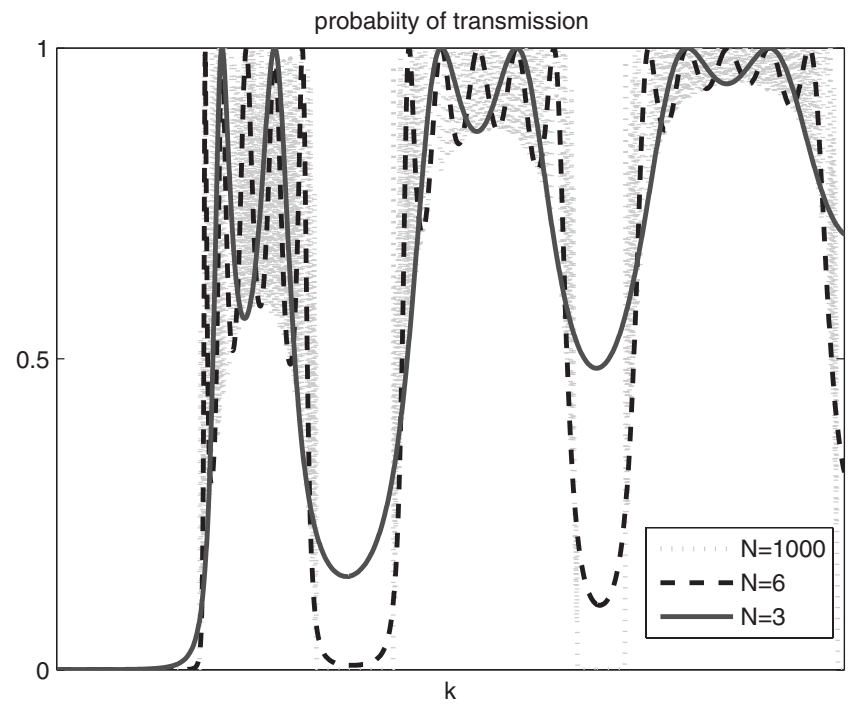

Fig. 4. Probability of transmission versus wave vector for a periodic array consisting of $N$ delta functions. Inside of the allowed bands, the probability of transmission is nonzero, with $N-1$ peaks where complete transmission is achieved. Outside of the band, total reflection occurs. As the wave vector increases, the width of the bands increases, and the width of the band gaps decreases.
Using Bloch's theorem and periodic boundary conditions, Ref. 4, p. 148, found the condition for being inside the band to be

$$
\frac{\cos (k a+\delta)}{|t|}=\cos q a,
$$

where $q$ is defined to be the crystal momentum of Bloch's theorem, $\delta$ is the relative phase gained by an electron by transmitting through a single barrier, and $k a$ is the geometric phase gained by an electron with wave vector $k$ that travels a distance $a$ between barriers. In Appendix B the total phase of the electron after encountering one barrier is defined as $\theta=k a+\delta$. This relation has a physically meaningful solution only when the magnitude of the left side of the equation is less than or equal to one, making the condition for being inside the band to be

$$
\frac{|\cos \theta|}{|t|}=\cos q a \leq 1 \text {. }
$$

Equations (16) and (18) are almost identical. The sole exception is that the former puts $|\cos (\theta)| /|t|=1$, corresponding to a crystal momentum of $q=0$, outside the band, whereas the latter puts it inside of the band, giving $N$ allowed states inside each band. This difference highlights the subtle but very real difference between the idealized crystal with periodic boundary conditions typically used to solve these problems and a real crystal that has boundaries with the outside world. This difference can be interpreted in several different ways. The energy of an electron with crystal momentum $q=0$ is $E=\hbar^{2} q^{2} / 2 m=0$. An electron with no energy has no momentum and therefore does not travel. There would be no way to perform a scattering experiment with such an electron because it would never enter the real crystal in a finite amount of time, even though in the idealized crystal its energy is inside the allowed band. In the idealized crystal, the application of periodic boundary conditions means that there is no such place as outside the crystal, and thus entering or leaving the crystal has no meaning. We can also resolve this discrepancy by considering the case of a single potential barrier with no periodicity. In this case the probability of transmission is given by $T=|t|^{2}$, and there are no energy states in which complete transmission occurs, except in the trivial case in which $T=1$, meaning the potential has no effect on the electron. Only upon the addition of another barrier are there energy states with zero resistance. In effect, the first potential in a periodic array of potentials represents the boundary between the inside and the outside of the crystal.

\section{DISORDERED SYSTEM}

An attractive feature of our method is that it can be easily modified to deal with disorder. Consider a system composed of individual potentials and thus still quasiperiodic but containing impurities with slightly different transmission and reflection coefficients. The addition of random impurities means that the system is no longer symmetric, and hence we can no longer assume that the reflection coefficient for electrons incident from the left, $\widetilde{r}_{N l}$, is the same as the reflection coefficient for electrons incident from the right, $\widetilde{r}_{N r}$. The derivation in Sec. III assumed that $\widetilde{r}_{N l}=\widetilde{r}_{N r}$; thus Eqs. (9) and (10) no longer apply. By rearranging the system we can derive similar equations that do apply. Figure 5 shows a system in which new potentials that are not necessarily identical are 
periodic potential

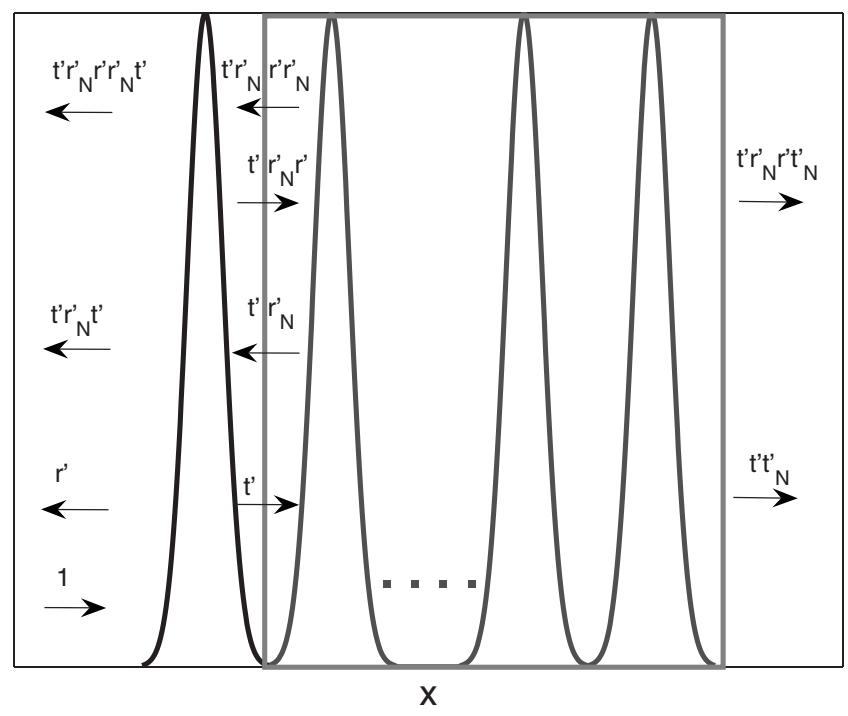

Fig. 5. An electron is incident upon a periodic potential composed of $N+1$ nonidentical potentials. Because the reflection coefficient for a disordered system can differ depending on whether an incoming wave is incident from the left or the right, the new potential is added at the beginning of the chain so that reflections only occur off of the $N$ original potentials (enclosed in the gray box) from the left. The new potential has reflections from the left and the right.

added at the beginning of the chain instead of at the end. In this case, incident waves are only reflected off of the chain of $N$ potentials from the right and never from the left. The transmission coefficient does not depend on the direction of incidence, which may be proved by reciprocity (see Ref. 5, p. 123). The following equations can be derived, similarly to Eqs. (9) and (10):

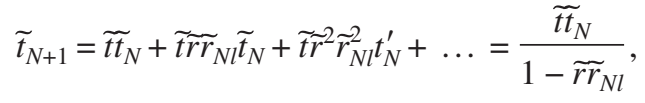

$$
\begin{aligned}
& \tilde{r}_{N+1 l}=\tilde{r}+\widetilde{t}^{2} \tilde{r}_{N l}+\widetilde{t}^{2} \tilde{r}_{N l}^{2} \tilde{r}+\ldots=\tilde{r}+\frac{\widetilde{t}^{2} \widetilde{r}_{N l}}{1-\widetilde{r r}_{N l}},
\end{aligned}
$$

where $\tilde{t}$ and $\tilde{r}$ are the transmission and reflection coefficients of the new potential to be added to the chain. Equations (9) and (19) are the same, and therefore in the absence of randomness, the transmission coefficient is the same no matter which side the potential is approached from. However Eqs. (10) and (20) are not identical.

Figure 6 shows the probability of transmission $T$ of an electron for a disordered system created by randomly placing two different potential barriers for the classical and quantum case. (Similar methods have been used in Ref. 6 to treat randomness in dimer models.) For the quantum case, the values of the total transmission coefficient for the two different barriers are $\tilde{t}_{1}=0.9 e^{0.56 i}$ and $\tilde{t}_{2}=0.6 e^{0.97 i}$. This pair of values was chosen because both give the same value for $q a$ in Eq. (18). In the classical case the same transmission probabilities were used, $T_{1}=0.81$ and $T_{2}=0.36$. In both cases, the probability of transmission approaches zero as the number of potential barriers increases. This increase means that in contrast to the completely ordered system, even electrons in the band are localized rather than having their wave functions extended throughout the entire potential. The length scale

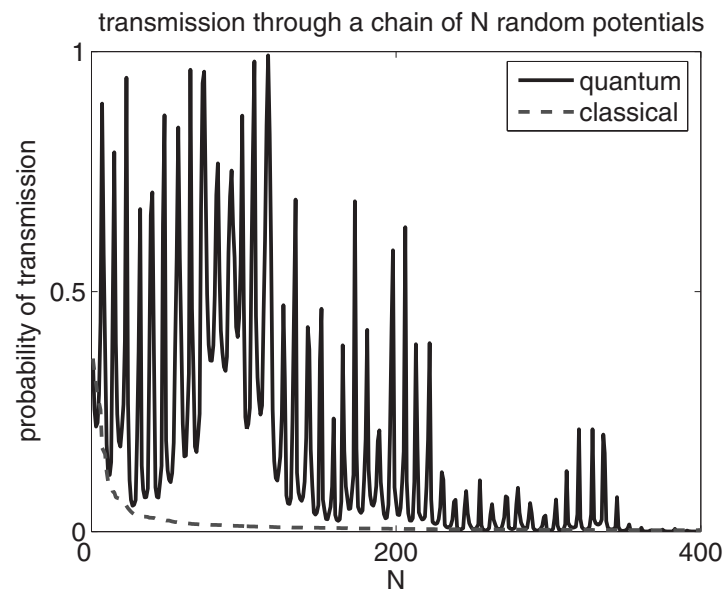

Fig. 6. Probability of transmission versus the number of random periodic potentials for the classical and quantum cases, with the energy in the latter case being inside a band. In both cases, two different potentials are used and their order is chosen randomly. In both cases the probability of transmission approaches zero.

over which the electron is likely to be found increases as $t_{1}^{\prime}$ and $t_{2}^{\prime}$ get closer together in phase and magnitude.

Figure 7 shows the effective resistance, $(1-T) / T$, of a disordered system created in the same manner. In the classical case the resistance grows linearly as discussed in Sec. II, whereas in the quantum case it grows exponentially. This exponential increase in resistance is in apparent violation of the linear dependence of resistance on length, which is generally observed macroscopically.

The solution to this paradox is to include the effects of temperature. The previous derivation, both for a perfectly periodic system or for a system with impurities, is valid only when the lattice of atomic cores remains in its equilibrium position. At $T=0$ the atomic cores remain fixed in place. The electron never undergoes a collision in which energy is exchanged between itself and an atomic core, and therefore its energy and hence the magnitude of the wave vector $k$ remain fixed. This assumption makes the derivation possible. Be-

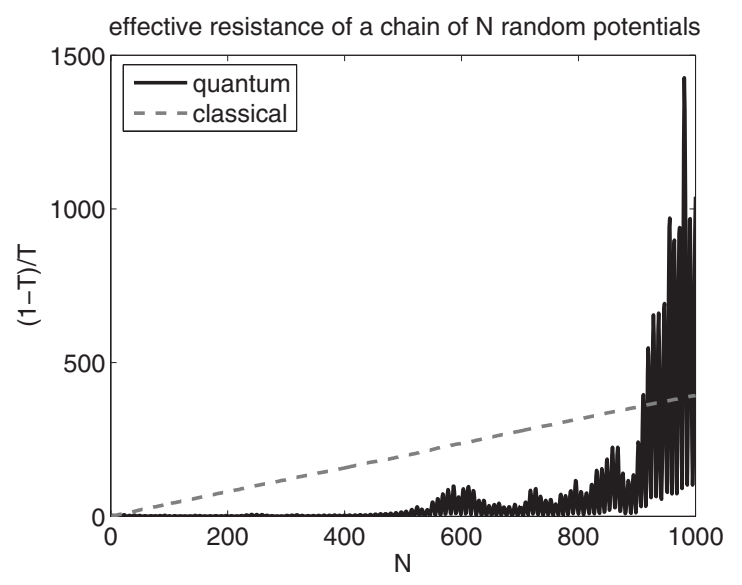

Fig. 7. Effective resistance versus the number of random periodic potentials for the classical and quantum cases, with the energy in the latter case being inside a band. In both cases, two different potentials are used and their order is chosen randomly. In both cases the resistance grows as the length of the chain increases; this increase is linear in the classical case and exponential in the quantum case. 
cause different paths through the potential have a fixed phase in relation to one another, constructive or destructive interference occurs.

Reality is different. Electrons can exchange energy with lattice vibrations, more so as the temperature increases. The energy exchange process constitutes a measurement. In principle, it would be possible to investigate the lattice before and after the collision, obtaining information about the electron. Just as in a double-slit experiment, quantum behavior (that is, interference) is observed only as long as the particular path the electron takes is not measured. We can define the mean free path $L_{c}(T)$ to be the average distance an electron travels between collisions, which exchange energy. Below this length, which is typically microscopic, quantum mechanics is needed to properly describe the electron's movement. Two contiguous sections of size $L_{c}$ in the sample behave like two incoherent scatterers, and their combined resistance is the sum of their resistances, as derived in Sec. II. Thus, we see that the resistance of a sample of length $L \gg L_{c}$ is proportional to $L$, in agreement with Ohm's law. In addition, because electrons are more likely to exchange energy with the lattice as the temperature increases, the resistance should increase linearly with temperature as is observed in metals near room temperature. As the temperature approaches zero, the resistance reaches zero (that is, a superconductor). If the electron scattered from every single atomic core, as suggested by the classical model, not only would the resistance be much larger than is observed, but also superconductivity would not be possible.

Our derivation has used a simple one-dimensional model to show how some nonclassical results arise as a result of the interference of the different possible paths of an electron. When more complete models are analyzed, a wider variety of results are found. In some circumstances, resistance decreases with temperature, or has a quadratic or exponential dependence. This richness of phenomena is what makes quantum mechanics such a fascinating field of study.

\section{ACKNOWLEDGMENTS}

This work was supported in part by the National Science Foundation under Grant No. DMR-0705460 and the U.S. Department of Energy, Office of Science, Office of Basic Energy Sciences, under Contract No. DE-AC02-06CH11357.

\section{APPENDIX A: RELATION BETWEEN TRANSMISSION AND REFLECTION COEFFICIENTS}

A free electron in the absence of a potential is described as a plane wave with wave function $\Psi=A e^{i k x}$, where $k$ is the wave vector. If the electron is incident upon a onedimensional potential of width $a$, the wave functions to the left and the right of the potential are

$$
\begin{aligned}
& \Psi_{l}=A e^{i k x}+r A e^{-i k x}, \\
& \Psi_{r}=t A e^{i k x} .
\end{aligned}
$$

For an electron incident from the right, the wave functions are given by

$$
\begin{aligned}
& \Psi_{r}=t D e^{-i k x}, \\
& \Psi_{l}=D e^{-i k x}+r D e^{i k x} .
\end{aligned}
$$

The transmission coefficient $t=|t| e^{i \delta}$ is assumed to be a known function of $k$. The reflection coefficient can be determined by writing these equations in matrix form with the wave functions to the left and right of the barrier given by

$$
\begin{aligned}
& \Psi_{l}=A e^{i k x}+B e^{-i k x}, \\
& \Psi_{r}=C e^{i k x}+D e^{-i k x} .
\end{aligned}
$$

Their coefficients are related by the $S$-matrix, which gives the relation of the outgoing waves to the incoming waves

$$
\left[\begin{array}{l}
B \\
C
\end{array}\right]=S\left[\begin{array}{l}
A \\
D
\end{array}\right], \quad S=\left[\begin{array}{ll}
r & t \\
t & r
\end{array}\right] .
$$

The $S$ matrix is unitary, which means that the magnitude of the vector of incoming waves is equal to the magnitude of the vector of outgoing waves, thus preserving the probability. The individual rows and columns of a unitary matrix each have length one, which means that $|t|^{2}+|r|^{2}=1$. The rows and columns of a unitary matrix are also orthogonal to one another, meaning that $r^{*} t+t^{*} r=0$. This condition is satisfied only if each term is purely imaginary $\operatorname{Re}\left(r^{*} t\right)=\operatorname{Re}\left(t^{*} r\right)=0$, meaning that

$$
\delta_{r}=\delta \pm \frac{\pi}{2}
$$

\section{APPENDIX B: TOTAL TRANSMISSION AND REFLECTION COEFFICIENTS}

The phase of the transmission coefficient $e^{i \delta}$ is the additional phase shift of the transmitted wave relative to a wave that has not encountered the potential barrier. A phase shift is also gained due to the fact that the wave has traveled a distance across the potential. This additional phase shift is hidden in the term $e^{i k x}$. If we are to correctly add waves that have undergone multiple transmissions and reflections in a periodic potential, thus traveling over different distances, the probability amplitude of the wave must be known explicitly. To do so, a coordinate transformation is performed with $x^{\prime}$ $=x+a / 2$ to the left of the barrier and $x^{\prime \prime}=x-a / 2$ to the right of the barrier. In the new coordinate system, $x^{\prime}$ gives the position relative to the left of the barrier, and $x^{\prime \prime}$ gives the position relative to the right of the barrier. Because the value of $A$ is arbitrary, we set to $e^{i k a / 2}$ for ease of calculations, and the wave functions are given by

$$
\begin{aligned}
& \Psi_{l}=e^{i k x^{\prime}}+r e^{i k a} e^{-i k x^{\prime}}, \\
& \Psi_{r}=t e^{i k a} e^{i k x^{\prime \prime}} .
\end{aligned}
$$

The total transmission and reflection coefficients, $\tilde{t}=t e^{i k a}=|t| e^{i \theta}$ and $\tilde{r}=r e^{i k a}= \pm i|r| e^{i \theta}$, now give the probability amplitude of each wave, with $\theta=k a+\delta$. Note that these new coefficients still satisfy the requirement of Eq. (A5). It can be seen that in addition to the phase $e^{i \delta}$, which is solely due to the potential, an additional geometric phase of $e^{i k a}$ is also gained upon both transmission and reflection, which reflects 
the distance traveled across the width of the potential. The relations $t=\widetilde{t} e^{-i k a}$ and $r=\widetilde{r} e^{-i k a}$ can be used to find the original transmission and reflection coefficients which give only the relative phase.

${ }^{1}$ L. de Broglie, "Theses de doctorat," Philos. Mag. 47, 446-458 (1924).

${ }^{2}$ G. P. Thomson and A. Reid, "Diffraction of cathode rays by a thin film," Nature (London) 119, 890-890 (1927).

${ }^{3}$ P. G. Merli, G. F. Missiroli, and G. Pozzi, "On the statistical aspect of electron interference phenomena," Am. J. Phys. 44, 306-307 (1976).

${ }^{4}$ N. W. Ashcroft and N. D. Mermin, Solid State Physics (Saunders, Philadelphia, 1976).

${ }^{5}$ S. Datta, Electronic Transport in Mesoscopic Systems (Cambridge U. P., Cambridge, 1995).

${ }^{6}$ D. H. Dunlap, H.-L. Wu, and P. W. Phillips, "Absence of localization in a random-dimer model,” Phys. Rev. Lett. 65 (1), 88-91 (1990).

${ }^{7}$ D. Kiang, "Multiple scattering by a Dirac comb," Am. J. Phys. 42, 785787 (1974).

${ }^{8}$ D. J. Griffiths and C. A. Steinke, "Waves in locally periodic media," Am. J. Phys. 69 (2), 137-154 (2001).

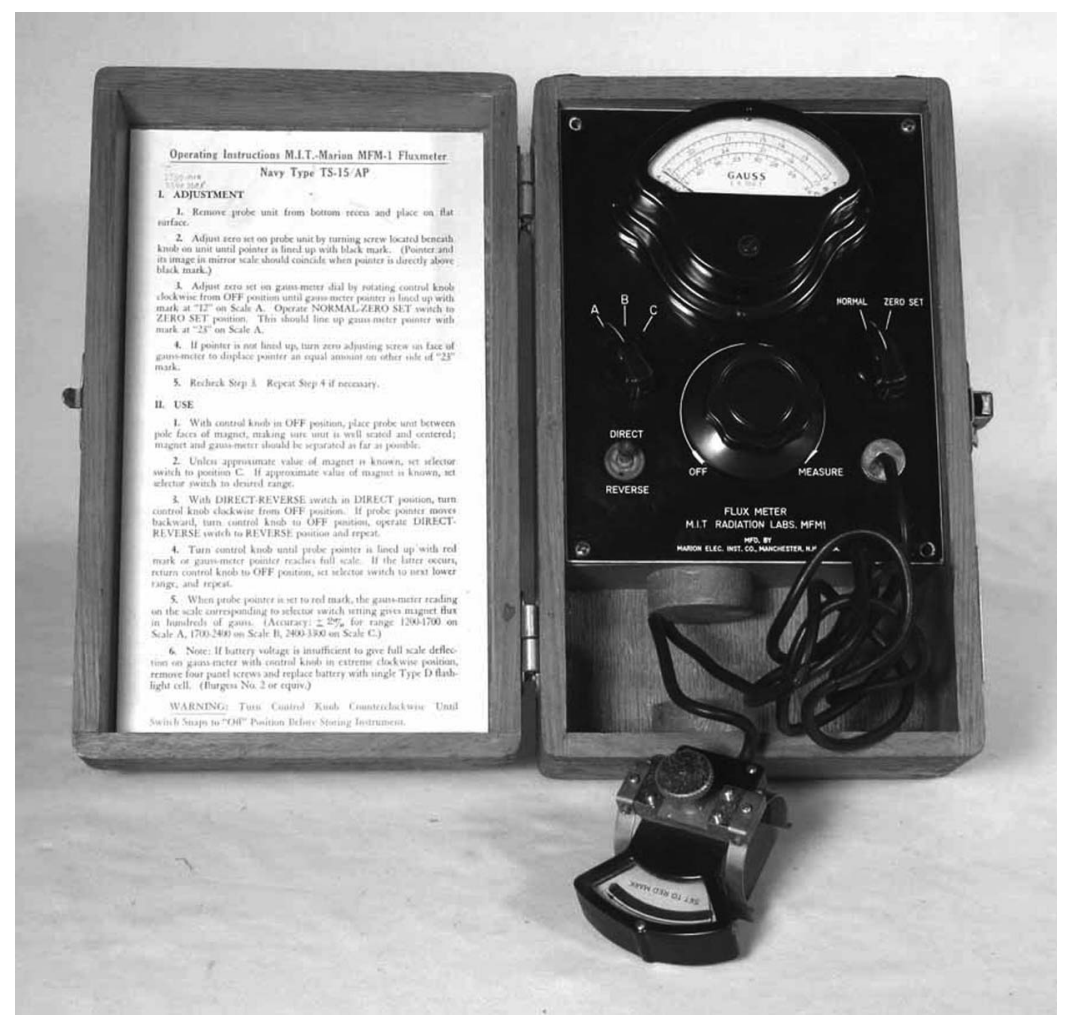

Flux meter. This instrument of World War II vintage was used at the Radiation Laboratory at MIT, and its operating manual, from August 1944, is marked Confidential on every page. Instructions for its destruction are included in the manual. It was used to measure the strength of the permanent magnets used with the magnetron tubes being developed at the Laboratory. The probe at the end of the cable is clipped onto the gap of the magnet. The meter movement of the probe coil is wound with high-purity silver wire and all of its other components are non-magnetic. The magnet under test acts as the source of the magnetic field for the D'Arsonval galvanometer that makes up the probe, and the resulting deflection of the meter with a standard current passing through it is a measure of the field. This instrument is in the Greenslade Collection. (Photograph and Notes by Thomas B. Greenslade, Jr., Kenyon College) 